\title{
PREFACE: ILASS 2020 SPECIAL ISSUE
}

A series of regional Institute for Liquid Atomization and Spray Systems (ILASS) conferences are held annually and focus on recent advances in experimental, computational, and theoretical analysis of the break-up process of liquids. The contributions cover a diverse range of fields including combustion systems, agricultural applications, fire suppression, and industrial processing sprays.

The 2020 ILASS-Americas conference was scheduled to be held in May of 2020 in Madison, WI. However, the conference was canceled due to the coronavirus disease (COVID-19) pandemic; therefore, papers submitted to ILASS-Americas 2020 were unable to be published in the conference proceedings and were not disseminated to the scientific community.

In a partnership with Atomization \& Sprays (the journal associated with ILASS) and Begell House, we are pleased to offer these special issues of Atomization \& Sprays, which collect journal papers based on conference submissions. ILASS-Americas 2020 authors were invited to submit to Atomization \& Sprays manuscripts that expanded on the papers submitted to the conference. The papers have undergone the normal peer-review process for the journal and meet the high standards of Atomization \& Sprays.

As Guest Editors, we are grateful to the authors for their contributions, and for their work to turn their conference papers into journal papers. In these special issues of the Atomization \& Sprays journal, the research advances unable to be shared at the canceled ILASS-Americas 2020 conference will be shared with the community, furthering the understanding of atomization and spray systems.

\section{Guest Editors:}

Mark Owkes, Montana State University, Bozeman, Montana 59715, USA, mark.owkes@montana.edu
Kyle M. Bade, Spraying Systems Co., Glendale Heights, Illinois 60139, USA, kyle.bade@spray.com 